\title{
PERTURBATIONS OF SOLUTIONS OF STIELTJES INTEGRAL EQUATIONS
}

\author{
BY \\ DAVID LOWELL LOVELADY
}

\begin{abstract}
Using multiplicative integration in two ways, formulae for solutions to perturbed Stieltjes integral equations are found in terms of unperturbed solutions. These formulae are used to obtain bounds on the difference between the perturbed solution and the unperturbed solution. The formulae are also used to explicitly solve, in terms of product integrals, a linear equation subject to nonlinear interface conditions.
\end{abstract}

I. Introduction. Let $X$ be a Banach space with norm $N_{1}$. We propose to study relationships between the solutions $f$ and $g$ of $f(t)=p+(R) \int_{t}^{c} d F[f]$ and $g(t)=$ $p+(R) \int_{t}^{c} d G[g]$, where $p$ is in $X, c$ is a real number, and $F$ and $G$ are functions from $[c, \infty)$ into a space of Lipschitz operators on $X$. J. W. Neuberger [9] has studied the first equation with $F$ continuous, and J. S. Mac Nerney [6] has extended this study to the case in which $F$ is of bounded variation. For the case in which the equations are equivalent to linear differential equations in matrix rings, C. C. Ross, Jr. [10] has obtained some multiplicative relationships for the solutions. Gantmacher [2, Chapter 14, §5] has also studied this case. R. H. Martin, Jr. [8] (see also [7]) has obtained bounds for solutions. We shall extend the work of Ross [10] to obtain formulae for the solutions of perturbed equations, and then use our formulae to obtain comparison bounds for perturbed solutions.

II. Preliminary results. Let $H$ be the set to which $A$ belongs only in case $A$ is a function from $X$ to $X, A(0)=0$, and there is a number $b$ so that $N_{1}[A(p)-A(q)]$ $\leqq b N_{1}[p-q]$ whenever $(p, q)$ is in $X \times X$. If $A$ is in $H$, let $N_{2}[A]$ be the least number $b$ so that $N_{1}[A(p)-A(q)] \leqq b N_{1}[p-q]$ whenever $(p, q)$ is in $X \times X$, and let $N_{3}[A]$ be the least number $b$ so that $N_{1}[A(p)] \leqq b N_{1}[p]$ whenever $p$ is in $X$.

Let $c$ be a real number, and let $S=[c, \infty)$. Let $O A$ be the set to which $V$ belongs only in case $V$ is a function from $S \times S$ to $H$ so that if $(x, y, z)$ is in $S \times S \times S$ and $z$ is between $x$ and $y$, then

(i) $V(x, z)+V(z, y)=V(x, y)$, and

(ii) there is a number $M$ so that

$$
\sum_{k=1}^{n} N_{2}\left[V\left(t_{k-1}, t_{k}\right)\right] \leqq M \quad \text { whenever }\left(t_{k}\right)_{k=0}^{n} \text { subdivides }(x, y) .
$$

Received by the editors February 12, 1970 and, in revised form, June 1, 1970.

AMS 1969 subject classifications. Primary 3453; Secondary 3495.

Key words and phrases. Perturbations, product integrals, bounds, stability, interface conditions. 
Let $O M$ be the set to which $W$ belongs only in case $W$ is a function from $S \times S$ to $H$ so that whenever $(x, y, z)$ is in $S \times S \times S$ and $z$ is between $x$ and $y$, then

(i) $W(x, z) W(z, y)=W(x, y)$, where the multiplication is by composition, and

(ii) there is a number $M$ so that

$$
\sum_{k=1}^{n} N_{2}\left[W\left(t_{k-1}, t_{k}\right)-I\right] \leqq M \quad \text { whenever }\left(t_{k}\right)_{k=0}^{n} \text { subdivides }(x, y),
$$

where $I$ in $H$ is given by $I(p)=p$.

Let $B V H$ be the set to which $f$ belongs only in case $f$ is a function from $S$ to $H$, of bounded $N_{2}$-variation on each bounded interval of $S$. Let $B V X$ be the set to which $f$ belongs only in case $f$ is a function from $S$ to $X$, of bounded $N_{1}$-variation on each bounded interval of $S$. If $F$ is a function from $S$ to either $H$ or $X$, let $d F$ be given on $S \times S$ by $d F(x, y)=F(y)-F(x)$.

Let $h$ be a function from $S \times S$ to $H, f$ a function from $S$ to $H$, and $g$ a function from $S$ to $H$. If $(a, b)$ is in $S \times S$, and $p$ is in $X$, by ${ }_{a} \Sigma^{b} h[p],{ }_{a} \Pi^{b} h[p],(L) \int_{a}^{b} f h[p]$, $(R) \int_{a}^{b} h g[p]$, and $(L, R) \int_{a}^{b} f h g[p]$ we mean the limit, in the sense of successive refinements of subdivisions, of members of $X$ of the forms $\sum_{k=1}^{n} h\left(t_{k-1}, t_{k}\right)[p]$, $\prod_{k=1}^{n} h\left(t_{k-1}, t_{k}\right)[p], \quad \sum_{k=1}^{n} f\left(t_{k-1}\right)\left[h\left(t_{k-1}, t_{k}\right)[p]\right], \quad \sum_{k=1}^{n} h\left(t_{k-1}, t_{k}\right)\left[g\left(t_{k}\right)[p]\right], \quad$ and $\sum_{k=1}^{n} f\left(t_{k-1}\right)\left[h\left(t_{k-1}, t_{k}\right)\left[g\left(t_{k}\right)[p]\right]\right]$, respectively, where $\left(t_{k}\right)_{k=0}^{n}$ is a monotone sequence into $S$ so that $a=t_{0}$ and $b=t_{n}$.

Our first three lemmas have proofs almost identical to the proofs of Lemmas 4.1, 4.3 , and 4.4 of [5], and we shall not exhibit these proofs here.

LEMMA 1. Let each of $f$ and $g$ be in BVH, and suppose each value of $f$ is linear. Let $(a, b)$ be in $S \times S$, and let $p$ be in $X$. Then if either of

$$
\text { (L) } \int_{a}^{b} f d g[p] \text { and }(R) \int_{a}^{b}(d f) g[p]
$$

exists, so also does the other, and

$$
(L) \int_{a}^{b} f d g[p]=f(b)[g(b)[p]]-f(a)[g(a)[p]]-(R) \int_{a}^{b}(d f) g[p] .
$$

LEMMA 2. If $f, g, a, b$, and $p$ are as in Lemma 1 , then $(L) \int_{a}^{b} f d g[p]$ exists.

Lemma 3. Let $V$ be in $O A$, and let each of $f, g, F$, and $G$ be in $B V H$, with each value of $f$ linear. Suppose that whenever $(a, b)$ is in $S \times S$, and $p$ is in $X$, then

$$
d F(a, b)[p]=(L) \int_{a}^{b} f V[p] \text { and } d G(a, b)[p]=(R) \int_{a}^{b} V g[p] .
$$

Then whenever $(a, b)$ is in $S \times S$, and $p$ is in $X$,

$$
(R) \int_{a}^{b}(d F) g[p]=(L, R) \int_{a}^{b} f V g[p]=(L) \int_{a}^{b} f d G[p] .
$$

REMARK. The existence of $(R) \int_{a}^{b} V g[p]$ was shown by Mac Nerney in [6]. 
LEMMA 4. Let $\left(A_{k}\right)_{k=1}^{n}$ and $\left(B_{k}\right)_{k=1}^{n}$ be sequences into H. Let $\left(a_{k}\right)_{k=1}^{n},\left(b_{k}\right)_{k=1}^{n}$, and $\left(c_{k}\right)_{k=1}^{n}$ be real-valued sequences so that, whenever $1 \leqq k \leqq n, N_{3}\left[A_{k}\right] \leqq a_{k}, N_{2}\left[B_{k}\right] \leqq b_{k}$, and $N_{3}\left[A_{k}-B_{k}\right] \leqq c_{k}$. Then

$$
N_{3}\left[\prod_{k=1}^{n} A_{k}-\prod_{k=1}^{n} B_{k}\right] \leqq \sum_{k=1}^{n}\left[\prod_{j=1}^{k-1} b_{j}\right] c_{k}\left[\prod_{j=k+1}^{n} a_{j}\right] .
$$

Proof.

$$
\begin{aligned}
N_{3}\left[\prod_{k=1}^{n} A_{k}-\prod_{k=1}^{n} B_{k}\right] & =N_{3}\left[\left(A_{1}-B_{1}\right) \prod_{k=2}^{n} A_{k}+B_{1} \prod_{k=2}^{n} A_{k}-B_{1} \prod_{k=2}^{n} B_{k}\right] \\
& \leqq c_{1} \prod_{k=2}^{n} a_{k}+b_{1} N_{3}\left[\prod_{k=2}^{n} A_{k}-\prod_{k=2}^{n} B_{k}\right] .
\end{aligned}
$$

The remainder of the proof is now clear.

LEMMA 5. Let $\left(A_{k}\right)_{k=1}^{n}$ and $\left(B_{k}\right)_{k=1}^{n}$ be sequences into H. Let $\left(a_{k}\right)_{k=1}^{n},\left(b_{k}\right)_{k=1}^{n}$, and $\left(c_{k}\right)_{k=1}^{n}$ be real-valued sequences so that, whenever $1 \leqq k \leqq n, N_{2}\left[A_{k}\right] \leqq a_{k}, N_{3}\left[B_{k}\right] \leqq b_{k}$, and $N_{3}\left[B_{k}-I\right] \leqq c_{k}$. Then

$$
N_{3}\left[\prod_{k=1}^{n} A_{k} B_{k}-\prod_{k=1}^{n} A_{k}\right] \leqq\left(\prod_{k=1}^{n} a_{k}\right) \sum_{k=1}^{n}\left[c_{k}\left(\prod_{j=k+1}^{n} b_{j}\right)\right] .
$$

REMARK. Lemma 5 is proved in much the same fashion as Lemma 4 , so we shall not prove it here.

In [6, Theorem 1.1], Mac Nerney showed that if $V$ is in $O A, W$ is in $O M,(a, b)$ is in $S \times S$, and $p$ is in $X$, then each of $a_{a} \Pi^{b}[I+V][p]$ and ${ }_{a} \sum^{b}[W-I][p]$ exists. Let $E$ be the function on $O A$, each value of which is a function from $S \times S$ to $H$, such that if $V$ is in $O A, E[V]$ is given by $E[V](a, b)[p]={ }_{a} \Pi^{b}[I+V][p]$. Then [6, Theorem 1.1] the range of $E$ is $O M, E$ is a bijection between $O A$ and $O M$, and if $V$ is in $O A, W=E[V]$, then $V(a, b)[p]={ }_{a} \sum^{b}[W-I][p]$ whenever $(a, b)$ is in $S \times S$ and $p$ is in $X$. Mac Nerney also showed [6, Corollary 2.1] that if $V$ is in $O A$, $W=E[V]$, and $h$ is given by $h(t)=W(t, c)$, then $h$ is in $B V H$, and is the only member of $B V H$ such that, if $p$ is in $X$,

$$
h(t)[p]=p+(R) \int_{t}^{c} V h[p]
$$

for each $t$ in $S$.

In [4], Herod showed that if each value of $V$, in $O A$, is linear, and $W=E[V]$, then each value of $W$ has inverse in $H$ only in case each of $I+V\left(t, t^{+}\right), I+V\left(t, t^{-}\right)$, $I+V\left(t^{+}, t\right)$, and $I+V\left(t^{-}, t\right)$ has inverse in $H$ whenever $t$ is in $S$. From this it is clear that $W(a, b)$ has inverse in $H$ whenever $a \geqq b$ only in case each of $I+V\left(t, t^{-}\right)$ and $I+V\left(t^{+}, t\right)$ has inverse in $H$ whenever $t$ is in $S$. Let $O A I$ be that subset of $O A$ to which $V$ belongs only in case each value of $V$ is linear, and each of $I+V\left(t, t^{-}\right)$ and $I+V\left(t^{+}, t\right)$ has inverse in $H$ whenever $t$ is in $S$. Another consequence of [4] 
is that if $V$ is in $O A I$, then $W(, c)^{-1}$ is in $B V H$. We shall need this last fact to ensure the existence of certain integrals.

III. A variation of parameters approach. If $V_{1}$ and $V_{2}$ are in $O A$, we can, in some circumstances, find a member $g$ of $B V H$ so that

$$
E\left[V_{1}+V_{2}\right](t, c)=E\left[V_{1}\right](t, c)[g(t)]
$$

for each $t$ in $S$.

THEOREM 1. Let $V_{1}$ be in $O A I, V_{2}$ in $O A$, and $W_{1}=E\left[V_{1}\right]$. Let $U$ be given by $U(a, b)[p]=(L, R) \int_{a}^{b} W_{1}(, c)^{-1} V_{2} W_{1}(, c)[p]$. Then

$$
E\left[V_{1}+V_{2}\right](t, c)[p]=W_{1}(t, c)[E[U](t, c)[p]],
$$

for each $t$ in $S$ and $p$ in $X$.

THEOREM 2. Let $V_{1}$ be in $O A I, V_{2}$ in $O A, W_{1}=E\left[V_{1}\right]$, and $W_{2}=E\left[V_{2}\right]$. Let $U$ be given by $U(a, b)[p]=(L, R) \int_{a}^{b} W_{1}(, c) V_{2} W_{1}(, c)^{-1}[p]$. Then

$$
W_{1}(t, c)\left[W_{2}(t, c)[p]\right]=E\left[V_{1}+U\right](t, c)[p]
$$

whenever $t$ is in $S$ and $p$ is in $X$.

REMARK. Since Theorem 2 follows from Theorem 1 simply by interchanging the roles of $V_{2}$ and $U$, we shall prove only Theorem 1. It should be noted that Theorem 2 includes Theorem 1 of [10].

Proof of Theorem 1. Let $g$ be given by $g(t)=E[U](t, c)$, and let $h$ be given by $h(t)=W_{1}(t, c)[g(t)]$. Now if $t$ is in $S$ and $p$ is in $X$,

$$
\text { (R) } \begin{aligned}
\int_{t}^{c} V_{2} h[p] & =(L, R) \int_{t}^{c} W_{1}(, c) W_{1}(, c)^{-1} V_{2} W_{1}(, c) g[p] \\
& =(L, R) \int_{t}^{c} W_{1}(, c) U g[p] \\
& =-(L) \int_{t}^{c} W_{1}(, c) d g[p] \\
& =(R) \int_{t}^{c}\left(d\left[W_{1}(, c)\right]\right) g[p]-p+W_{1}(t, c)[g(t)[p]] \\
& =-(R) \int_{t}^{c} V_{1} W_{1}(, c) g[p]-p+h(t)[p] \\
& =-(R) \int_{t}^{c} V_{1} h[p]-p+h(t)[p] .
\end{aligned}
$$

This completes the proof.

The remainder of $\S I I I$ is devoted to exploring the consequences of Theorem 2 in the case in which each of $V_{1}$ and $V_{2}$ is in $O A I$. Our next result follows immediately. 
Corollary 1. Let each of $V_{1}$ and $V_{2}$ be in $O A I$, with $W_{1}=E\left[V_{1}\right]$. Let $U$ be given by

$$
U(a, b)[p]=(L, R) \int_{a}^{b} W_{1}(, c) V_{2} W_{1}(, c)^{-1}[p]
$$

Then $V_{1}+U$ is in $O A I$.

Now we produce a necessary and sufficient condition for commutativity. Our result includes Theorem 2 of [10].

THEOREM 3. Let each of $V_{1}$ and $V_{2}$ be in $O A I$, with $W_{1}=E\left[V_{1}\right]$ and $W_{2}=E\left[V_{2}\right]$. Then these are equivalent:

$$
\begin{aligned}
& W_{1}(t, c) W_{2}(t, c)=W_{2}(t, c) W_{1}(t, c) \text { for each } t \text { in } S . \\
& V_{1}(a, b)[p]-V_{2}(a, b)[p] \\
& \quad=(L, R) \int_{a}^{b}\left[W_{2}(, c) V_{1} W_{2}(, c)^{-1}[p]-W_{1}(, c) V_{2} W_{1}(, c)^{-1}[p]\right]
\end{aligned}
$$

whenever $p$ is in $X$ and $a \geqq b$.

Proof. Choose $U_{1}$ and $U_{2}$, according to Theorem 2, so that if $t$ is in $S$,

$$
W_{1}(t, c) W_{2}(t, c)=E\left[V_{1}+U_{1}\right](t, c) \quad \text { and } \quad W_{2}(t, c) W_{1}(t, c)=E\left[V_{2}+U_{2}\right](t, c) \text {. }
$$

Now (i) holds only in case $E\left[V_{1}+U_{1}\right](t, c)=E\left[V_{2}+U_{2}\right](t, c)$ for each $t$ in $S$. Since each of $V_{1}+U_{1}$ and $V_{2}+U_{2}$ is in $O A I$, this is equivalent to $E\left[V_{1}+U_{1}\right](a, b)$ $=E\left[V_{2}+U_{2}\right](a, b)$ whenever $a \geqq b$, which in turn is equivalent to $V_{1}(a, b)-V_{2}(a, b)$ $=U_{2}(a, b)-U_{1}(a, b)$ whenever $a \geqq b$, which is (ii). The proof is complete.

An interesting question is: When does $E$ preserve the exponential identity on $O A I$ ? We answer this question with a theorem which includes Theorem 3 of [10].

THEOREM 4. Let each of $V_{1}$ and $V_{2}$ be in $O A I$, with $W_{1}=E\left[V_{1}\right]$ and $W_{2}=E\left[V_{2}\right]$. Then these are equivalent:

(i) $W_{1}(t, c) W_{2}(t, c)=E\left[V_{1}+V_{2}\right](t, c)$ for each $t$ in $S$.

(ii) $V_{2}(a, b)[p]=(L, R) \int_{a}^{b} W_{1}(, c) V_{2} W_{1}(, c)^{-1}[p]$ for $a \geqq b$ and $p$ in $X$.

Remark. Theorem 4 follows immediately from Theorem 2 and Corollary 1 , so we shall not prove it here.

An interesting special case in the multiplication of solutions comes in taking powers. We now handle that case.

THeOrem 5. Let $V$ be in $O A I$, and let $W=E[V]$. Then these are equivalent:

(i) $E[k V](t, c)=[W(t, c)]^{k}$ for each $t$ in $S$ and each positive integer $k$.

(ii) $V(a, b)[p]=(L, R) \int_{a}^{b} W(, c) V W(, c)^{-1}[p]$ for $a \geqq b$ and $p$ in $X$.

IV. An integrated multiplication approach. If $V_{1}$ and $V_{2}$ are in $O A$, we can, under certain circumstances, find a formula for $E\left[V_{1}+V_{2}\right]$ in terms of products of $E\left[V_{1}\right]$ and $E\left[V_{2}\right]$. 
THEOREM 6. Let each of $V_{1}$ and $V_{2}$ be in $O A$, with $W_{1}=E\left[V_{1}\right]$ and $W_{2}=E\left[V_{2}\right]$. Suppose that whenever $(a, b)$ is in $S \times S$, then ${ }_{a} \sum^{b} N_{3}\left[V_{1}\left[I+V_{2}\right]-V_{1}\right]=0$. Then whenever $(a, b)$ is in $S \times S$ and $p$ is in $X,{ }_{a} \Pi^{b} W_{1} W_{2}[p]$ exists. Furthermore, if $W_{3}$ is given by $W_{3}(a, b)[p]={ }_{a} \prod^{b} W_{1} W_{2}[p]$, then $W_{3}=E\left[V_{1}+V_{2}\right]$.

Remark. Helton $[3, \S 5]$ has studied this kind of integrated multiplication in connection with linear equations.

Proof. Let $(a, b)$ be in $S \times S$ and $p$ in $X$. It is clear that ${ }_{a} \Pi^{b} W_{1} W_{2}[p]$, if it exists, is given by ${ }_{a} \Pi^{b}\left[I+V_{1}\right]\left[I+V_{2}\right][p]$. Let $\left(t_{k}\right)_{k=0}^{n}$ be a partition of $(a, b)$. Now by Lemma 4 ,

$$
\begin{gathered}
N_{1}\left[\prod_{k=1}^{n}\left[I+V_{1}\left(t_{k-1}, t_{k}\right)\right]\left[I+V_{2}\left(t_{k-1}, t_{k}\right)\right][p]\right. \\
\left.-\prod_{k=1}^{n}\left[I+V_{1}\left(t_{k-1}, t_{k}\right)+V_{2}\left(t_{k-1}, t_{k}\right)\right][p]\right] \\
\leqq N_{1}[p] \prod_{k=1}^{n}\left[1+N_{2}\left[V_{1}\left(t_{k-1}, t_{k}\right)\right]\right]\left[1+N_{2}\left[V_{2}\left(t_{k-1}, t_{k}\right)\right]\right] \\
\quad \sum_{k=1}^{n} N_{3}\left[V_{1}\left(t_{k-1}, t_{k}\right)\left[I+V_{2}\left(t_{k-1}, t_{k}\right)\right]-V_{1}\left(t_{k-1}, t_{k}\right)\right] .
\end{gathered}
$$

The proof is now clear.

We can use this theorem, and the following lemma, to get two companion theorems to Theorem 5 .

LEMMA 6. If $A$ is in $H$, and $n$ is a positive integer, then

$$
[I+A]^{n}=I+A+\sum_{k=1}^{n-1} A[I+A]^{k} .
$$

Consequently,

$$
[I+A]^{n}-[I+n A]=\sum_{k=1}^{n-1}\left[A[I+A]^{k}-A\right] .
$$

THEOREM 7. Let $V$ be in $O A, W=E[V]$, and let $n$ be a positive integer. Suppose that whenever $(a, b)$ is in $S \times S$, and $k$ is an integer, $1 \leqq k \leqq n$, then

$$
{ }_{a} \sum^{b} N_{3}\left[V[I+V]^{k}-V\right]=0 .
$$

Then if $(a, b)$ is in $S \times S$ and $p$ is in $X, \Pi^{b} W^{n}[p]$ exists. Furthermore, if $M$ is given by $M(a, b)[p]={ }_{a} \Pi^{b} W^{n}[p]$, then $M=E[n V]$.

THEOREM 8. Let $V$ be in $O A, W=E[V]$, and suppose each value of $V$ is linear. Suppose that if $(a, b)$ is in $S \times S$, then ${ }_{a} \Sigma^{b} N_{3}\left[V^{2}\right]=0$. Then if $k$ is a positive integer, $(a, b)$ is in $S \times S$, and $p$ is in $X$, then

$$
{ }_{a} \prod^{b} W^{k}[p]={ }_{a} \prod^{b}[I+k V][p] .
$$


Remarks. The hypotheses of Theorem 7 will be satisfied if $V$ is $N_{3}$-continuous. Herod, in [4], has shown that if each value of $V$ is linear, and ${ }_{a} \sum^{b} N_{3}\left[V^{2}\right]=0$ for each $(a, b)$ in $S \times S$, then $V$ is in $O A I$. To prove Theorem 7 , imitate the proof of Theorem 6, and use Lemma 5. Theorem 8 follows quickly from Theorem 7.

V. The comparison of perturbed solutions. In differential equations, much use is made of the variation-of-parameters formula in the study of perturbed solutions. Our first result extends that idea.

LEMMA 7. Let $V_{1}$ be in $O A I, V_{2}$ in $O A$, and $W_{1}=E\left[V_{1}\right]$. Let $h$ be that member of $B V H$ such that if $(t, p)$ is in $S \times X$,

$$
h(t)[p]=p+(R) \int_{t}^{c} V_{1} h[p]+(R) \int_{t}^{c} V_{2} h[p] .
$$

Then if $(t, p)$ is in $S \times X$,

$$
h(t)[p]=W_{1}(t, c)[p]+(L, R) \int_{t}^{c} W_{1}(t,) V_{2} h[p] .
$$

Proof. Let $g$ be that member of $B V H$ such that if $(t, p)$ is in $S \times X$, then $g(t)[p]$ $=p+(L, R) \int_{t}^{c} W_{1}(, c)^{-1} V_{2} W_{1}(, c) g[p]$. Now by Theorem 1 we have $h(t)=$ $W_{1}(t, c) g(t)$ for each $t$ in $S$. Since each value of $W_{1}$ is linear, this says

$$
\begin{aligned}
h(t)[p] & =W_{1}(t, c)[p]+W_{1}(t, c) \cdot(L, R) \int_{t}^{c} W_{1}(, c)^{-1} V_{2} h[p] \\
& =W_{1}(t, c)[p]+(L, R) \int_{t}^{c} W_{1}(t, \quad) V_{2} h[p],
\end{aligned}
$$

whenever $(t, p)$ is in $S \times X$. This completes the proof.

Since $X$ is a Banach space, the set of real numbers can be considered as a subset of $H$. Let $O A R$ (respectively $O M R$ ) be the subset of $O A$ (respectively $O M$ ) which consists of real-valued functions. Martin, in [8] (see also [7]), has shown that if $V$ is in $O A$, and $(a, b)$ is in $S \times S$, then ${ }_{a} \Sigma^{b}\left(N_{3}[I+V]-1\right)$ exists. Furthermore, if $\gamma$ on $S \times S$ is given by $\gamma(a, b)={ }_{a} \sum^{b}\left(N_{3}[I+V]-1\right)$, then $\gamma$ is in $O A R$. Also, if $W=E[V]$ and $\lambda=E[\gamma]$, then $\lambda$ is the least member of $O M R$ such that $N_{3}[W(a, b)] \leqq \lambda(a, b)$ for each $(a, b)$ in $S \times S$, i.e., $\lambda(a, b)={ }_{a} \Pi^{b} N_{3}[W]$ [7, Theorems 3.2 and 3.3] (see also [6, Theorems 1 and 2]).

LEMMA 8. Let each of $V_{1}$ and $V_{2}$ be in $O A, W_{1}=E\left[V_{1}\right]$, and $W_{2}=E\left[V_{2}\right]$. For $i=1,2$, let $\gamma_{i}$ be given by $\gamma_{i}(a, b)={ }_{a} \sum^{b}\left(N_{3}\left[I+V_{i}\right]-1\right)$, and let $\lambda_{i}=E\left[\gamma_{i}\right]$. Let $\gamma_{3}$ be given by $\gamma_{3}(a, b)={ }_{a} \sum^{b}\left(N_{3}\left[I+V_{1}+V_{2}\right]-1\right)$, and let $\lambda_{3}=E\left[\gamma_{3}\right]$. Suppose that whenever $(a, b)$ is in $S \times S$, then ${ }_{a} \sum^{b} N_{3}\left[V_{1}\left[I+V_{2}\right]-V_{1}\right]=0$ and ${ }_{a} \sum^{b}\left|\gamma_{1} \gamma_{2}\right|=0$. Then whenever $(a, b)$ is in $S \times S$,

and

$$
\gamma_{3}(a, b) \leqq \gamma_{1}(a, b)+\gamma_{2}(a, b),
$$

$$
\lambda_{3}(a, b) \leqq \lambda_{1}(a, b) \lambda_{2}(a, b)
$$


Proof. Let $W_{3}=E\left[V_{1}+V_{2}\right]$. Now, if $(a, b)$ is in $S \times S$,

$$
\begin{aligned}
& \lambda_{3}(a, b)={ }_{a} \prod^{b} N_{3}\left[W_{3}\right] \leqq \prod_{a}^{b} N_{3}\left[W_{1}\right] N_{3}\left[W_{2}\right]=\lambda_{1}(a, b) \lambda_{2}(a, b), \\
& \gamma_{3}(a, b)={ }_{a} \sum^{b}\left[\lambda_{3}-1\right] \leqq \sum_{a}^{b}\left[\lambda_{1} \lambda_{2}-1\right]=\gamma_{1}(a, b)+\gamma_{2}(a, b) .
\end{aligned}
$$

The proof is complete.

THEOREM 9. Let $V_{1}, V_{2}, \gamma_{1}$, and $\gamma_{2}$ be as in Lemma 8. Let $h$ be that member of $B V H$ such that

$$
h(t)[p]=p+(R) \int_{t}^{c} V_{1} h[p]+(R) \int_{t}^{c} V_{2} h[p]
$$

whenever $(t, p)$ is in $S \times X$, and let $F$ be that real-valued member of $B V H$ such that

$$
F(t)=1+(R) \int_{t}^{c} \gamma_{1} F+(R) \int_{t}^{c} \gamma_{2} F
$$

for each $t$ in $S$. Then $N_{3}[h(t)] \leqq F(t)$ for each $t$ in $S$.

REMARK. Note that if $\lambda_{1}=E\left[\gamma_{1}\right]$, and $\lambda_{1}$ is never zero, then, by Theorem 1, $F(t)=\lambda_{1}(t, c){ }_{t} \Pi^{c}\left[1+\lambda_{1}^{-1} \gamma_{2}\right]$ for each $t$ in $S$. Theorem 9 follows immediately from Lemma 8 and the aforementioned work of Martin [8] (see also [7]).

Now we obtain a comparison bound for a nonlinear perturbation of a linear equation. Our theorem follows directly from Theorem 9 and Lemma 7.

TheOREM 10. Let $V_{1}$ be in $O A I, V_{2}$ in $O A$, and $W_{1}=E\left[V_{1}\right]$. Let $\lambda_{1}$ and $\gamma_{2}$ be given by $\lambda_{1}(a, b)={ }_{a} \Pi^{b} N_{3}\left[W_{1}\right]$ and $\gamma_{2}(a, b)={ }_{a} \sum^{b}\left(N_{3}\left[V_{2}+I\right]-1\right)$. Let $\beta$ be $a$ member of $O A R$ such that $N_{3}\left[V_{2}(a, b)\right] \leqq \beta(a, b)$ for each $(a, b)$ in $S \times S$. Let $h$ in $B V H$ be given by $h(t)=E\left[V_{1}+V_{2}\right](t, c)$. Let $G$ be given by $G(t)={ }_{t} \Pi^{c}\left[1+\lambda_{1}^{-1} \gamma_{2}\right]$. Then if $t$ is in $S$,

$$
N_{3}\left[h(t)-W_{1}(t, c)\right] \leqq \lambda_{1}(t, c) \cdot(R) \int_{t}^{c} \lambda_{1}^{-1} \beta G
$$

REMARK. One might speculate on replacing $\beta$, in the conclusion of Theorem 10 , by $\gamma_{2}$. This, in general, is not possible since $\gamma_{2}$ may have negative values.

We can get a bound for the difference of solutions even when $V_{1}$ is not linear.

THEOREM 11. Let each of $V_{1}$ and $V_{2}$ be in $O A$, with $W_{1}=E\left[V_{1}\right]$ and $W_{2}=E\left[V_{2}\right]$. Suppose ${ }_{a} \sum^{b} N_{3}\left[V_{1}\left[I+V_{2}\right]-V_{1}\right]=0$ whenever $(a, b)$ is in $S \times S$. Let $\lambda_{1}, \beta$, and $\lambda_{2}$ be given on $S \times S$ by $\lambda_{1}(a, b)={ }_{a} \Pi^{b} N_{2}\left[W_{1}\right], \beta(a, b)={ }_{a} \sum^{b} N_{3}\left[V_{2}\right]$, and $\lambda_{2}(a, b)=$ ${ }_{a} \prod^{b} N_{3}\left[W_{2}\right]$. Then whenever $(a, b)$ is in $S \times S$,

$$
N_{3}\left[E\left[V_{1}+V_{2}\right](a, b)-W_{1}(a, b)\right] \leqq \lambda_{1}(a, b) \cdot(R) \int_{a}^{b} \beta \lambda_{2}(, b) .
$$

REMARK. It should be noted that $\lambda_{1}$ is given by products of $N_{2}\left[W_{1}\right]$, not $N_{3}\left[W_{1}\right]$ as before. Theorem 11 follows from Lemmas 4 and 5 . 
VI. Applications. We shall show that our results include a slight modification of a theorem of Coppel, [1, p. 64], and we shall obtain a bound for perturbations of nonlinear differential equations. We shall also use our results to solve both linear and nonlinear interface problems for Stieltjes integral equations. Interface problems have been studied by Stallard, [11] and [12], and Zettl, [13], for differential equations.

THeOrem 12. Let $V_{1}$ be in $O A I, V_{2}$ in $O A$, and $W_{1}=E\left[V_{1}\right]$. Suppose there are numbers $K$ and $M$ so that $N_{3}\left[W_{1}(t, s)\right] \leqq K$ and ${ }_{c} \sum^{t} N_{3}\left[V_{2}\right] \leqq M$ for $c \leqq s \leqq t$. Let $h=E\left[V_{1}+V_{2}\right](, c)$. Then if $\varepsilon>0$ there is $\delta>0$ so that whenever $p$ is in $X$ and $N_{1}[p]<\delta$, then $N_{1}[h(t)[p]]<\varepsilon$ for each $t$ in $S$. Furthermore, if $N_{3}-\lim _{t \rightarrow \infty} W_{1}(t, s)$ $=0$ for each $s$ in $S$, then $N_{1}-\lim _{t \rightarrow \infty} h(t)[p]=0$ for each $p$ in $S$.

RemarK. This theorem includes Coppel's theorem in the case in which the perturbation is Lipschitz.

Proof. From Lemma 7, $h(t)[p]=W_{1}(t, c)[p]+(L, R) \int_{t}^{c} W_{1}(t, \quad) V_{2} h[p]$ for each $(t, p)$ in $S \times X$. Let $\beta$ be given by $\beta(a, b)={ }_{a} \sum^{b} N_{3}\left[V_{2}\right]$. Let $(t, p)$ be in $S \times X$. Now

$$
N_{1}[h(t)[p]] \leqq K N_{1}[p]+(R) \int_{t}^{c} K \beta N_{1}[h()[p]]
$$

Let $Q$ be given on $S$ by

$$
Q(t)=K N_{1}[p]+(R) \int_{t}^{c} K \beta N_{1}[h()[p]]-N_{1}[h(t)[p]] .
$$

Now $Q$ has only nonnegative values and, if $t$ is in $S$,

$$
N_{1}[h(t)[p]]=K N_{1}[p]-Q(t)+(R) \int_{t}^{c} K \beta N_{1}[h()[p]] .
$$

Let $F$ be that nonnegative-valued member of $B V H$ so that

$$
F(t)=K N_{1}[p]+(R) \int_{t}^{c} K \beta F
$$

for each $t$ in $S$. Now each of $(*)$ and (**) can be solved by iteration [6, Theorem 2], and $-Q(t) \leqq 0$ for each $t$ in $S$, so $N_{1}[h(t)[p]] \leqq F(t)$ for each $t$ in $S$. But

$$
\begin{aligned}
F(t)={ }_{t} \prod^{c}[1+K \beta] K N_{1}[p] & \leqq(\exp [K \beta(t, c)]) K N_{1}[p] \\
& \leqq K N_{1}[p](\exp [K M])
\end{aligned}
$$

for each $t$ in $S$. This proves the first part of the theorem.

Note that in the first part of the proof we in fact showed that whenever $p$ is in $X$, then $h()[p]$ is bounded. Now let $p$ be in $S$. If $t \geqq s$, and $B$ is such that $N_{1}[h(r)[p]] \leqq B$ for each $r$ in $S$, then

$$
N_{1}\left[(R) \int_{t}^{s} W_{1}(t, \quad) V_{2} h[p]\right] \leqq K B \beta(t, s)
$$


Let $\varepsilon>0$ and find $s_{0}$ so that $\beta\left(t, s_{0}\right)<\varepsilon /(K B+1)$. Now if $t>s_{0}$,

$$
\begin{aligned}
N_{1}[h(t)[p]] \leqq & N_{3}\left[W_{1}(t, c)\right] N_{1}[p]+(L, R) \int_{s_{0}}^{c} N_{3}\left[W_{1}(t,)\right] \beta N_{1}[h()[p]]+\varepsilon \\
\leqq & N_{3}\left[W_{1}(t, c)\right] N_{1}[p] \\
& +N_{3}\left[W_{1}\left(t, s_{0}\right)\right] \cdot(L, R) \int_{s_{0}}^{c} N_{3}\left[W_{1}\left(s_{0}, \quad\right)\right] \beta N_{1}[h()[p]]+\varepsilon
\end{aligned}
$$

The proof is now clear.

Let $M$ be that real-valued function on $H$ given by

$$
M[A]=\lim _{t \rightarrow 0+}(1 / t)\left[N_{3}[I+t A]-1\right]
$$

Suppose $B$ is an $N_{2}$-continuous function from $S$ to $H$, and let $V$ be given by $V(s, t)=\int_{s}^{t} B(r) d r$, if $s \leqq t$, and $V(s, t)=V(t, s)$ if $t \leqq s$. Let $\gamma$ be given by $\gamma(a, b)$ $={ }_{a} \sum^{b}\left[N_{3}[I+V]-1\right]$. Martin, [8, Example 3.1] (see also [7]), has shown that if $s \leqq t$, then $\gamma(s, t)=\int_{s}^{t} M[B(r)] d r$ and $\gamma(s, t)=\gamma(t, s)$ if $t \leqq s$. Noting these results, we state without proof the following consequence of Theorem 11 .

THEOREM 13. Let each of $A$ and $B$ be an $N_{2}$-continuous function from $S$ to $H$. Let $W$ be that function from $S \times S$ to $H$ such that if $(s, p)$ is in $S \times X$, then $W(, s)[p]$ is the solution of $h^{\prime}(t)=A(t)[h(t)], h(s)=p$. Let $\lambda$ be given by $\lambda(a, b)={ }_{a} \Pi^{b} N_{2}[W]$. Let $p$ be in $X$, and let $h$ and $g$ be continuously differentiable members of $B V X$ such that $h(c)=g(c)=p, h^{\prime}(t)=A(t)[h(t)]+B(t)[h(t)]$, and $g^{\prime}(t)=A(t)[g(t)]$ whenever $t$ is in $S$. Then, if $t$ is in $S$,

$$
N_{1}[h(t)-g(t)] \leqq N_{1}[p] \lambda(t, c) \int_{c}^{t} N_{3}[B(s)] \exp \left[\int_{c}^{s} M[B(r)] d r\right] d s .
$$

In $[12, \S 3]$, Stallard showed how to solve the following problem: Let $P$ be a Lebesgue summable matrix-valued function on an interval $[a, b]$. Let $\left(A_{k}\right)_{k=1}^{n}$ be a matrix-valued sequence, and let $a<t_{1}<t_{2}<\cdots<t_{n}<b$. Find a matrix-valued function $Y$ on $[a, b]$ so that

(i) $Y(a)=I$, where $I$ is the identity matrix,

(ii) $Y\left(t_{k}^{+}\right)=A_{k} Y\left(t_{k}^{-}\right)$for $k=1,2, \ldots, n$,

(iii) $Y$ is absolutely continuous on $\left[a, t_{1}\right),\left(t_{n}, b\right]$, and $\left(t_{k-1}, t_{k}\right)$ for $k=2,3, \ldots, n$, and

(iv) $Y^{\prime}(t)=P(t) Y(t)$, almost everywhere in $\left(a, t_{1}\right),\left(t_{n}, b\right)$, and $\left(t_{k-1}, t_{k}\right)$ for $k=2,3, \ldots, n$.

We shall extend this problem to Stieltjes integral equations, and show that under certain circumstances we can write a formula for the solution.

THEOREM 14. Let $V$ be in $O A$, and let $K$ be a countable subset of $S, c$ not in $K$. Let each of $A$ and $B$ be a function from $K$ to $H$ such that if $M$ is a bounded subset of $K$, then each of $\sum_{t \text { in } M} N_{2}\left[A(t)-I-V\left(t, t^{-}\right)\right]$and $\sum_{t \text { in } M} N_{2}\left[B(t)-I-V\left(t^{+}, t\right)\right]$ is 
finite. Then whenever $p$ is in $X$ there is a member $h$ of $B V X$ such that each of (i), (ii), and (iii) is true.

(i) $h(c)=p$.

(ii) If $t$ is in $K$, then $h(t)=A(t)\left[h\left(t^{-}\right)\right]$and $h\left(t^{+}\right)=B(t)[h(t)]$.

(iii) If $N$ is an open connected subset of $S$ in the complement of $K$, then there is $q$ in $X$ so that

whenever $t$ is in $N$.

$$
h(t)=q+(R) \int_{t}^{c} V[h]
$$

ReMarK. It should be noted that, if $K$ has no limit point, the conditions on $A$ and $B$ are satisfied a priori. In this circumstance it is clear that $h$ is unique, and hence this theorem includes Stallard's result [12]. In other circumstances, additional conditions on $A, B$, and $K$ may be necessary to ensure uniqueness (see [11, pp. 65-66]).

Proof. Let $U_{1}$ and $U_{2}$ be members of $O A$ such that if $[a, b]$ is an interval in $S$ then $U_{1}(b, a)=0$ if $(a, b]$ does not intersect $K, U_{2}(b, a)=0$ if $[a, b)$ does not intersect $K$,

$$
U_{1}(b, a)=\sum_{s \text { in } K, a<s \leqq b}\left[A(s)-I-V\left(s, s^{-}\right)\right]
$$

if $(a, b]$ does intersect $K$, and

$$
U_{2}(b, a)=\sum_{s \ln K, a \leqq s<b}\left[B(s)-I-V\left(s^{+}, s\right)\right]
$$

if $[a, b)$ does intersect $K$.

Now if $t$ is in $K$,

$$
\begin{array}{ll}
U_{1}\left(t, t^{-}\right)=A(t)-I-V\left(t, t^{-}\right), & U_{1}\left(t^{+}, t\right)=0, \\
U_{2}\left(t, t^{-}\right)=0, & U_{2}\left(t^{+}, t\right)=B(t)-I-V\left(t^{+}, t\right) .
\end{array}
$$

Let $p$ be in $X$, and let $h$ be given by $h(t)=E\left[V+U_{1}+U_{2}\right](t, c)[p]$. Now $h$ is in $B V X$, and if $t$ is in $S$ then

$$
h(t)=p+(R) \int_{t}^{c} V[h]+(R) \int_{t}^{c} U_{1}[h]+(R) \int_{t}^{c} U_{2}[h] .
$$

Hence (i) and (iii) follow immediately. Also, if $t$ is in $S, t>c$, then

$$
h(t)=\left[I+V+U_{1}+U_{2}\right]\left(t, t^{-}\right)\left[h\left(t^{-}\right)\right]
$$

and

$$
h\left(t^{+}\right)=\left[I+V+U_{1}+U_{2}\right]\left(t^{+}, t\right)[h(t)] .
$$

Thus, if $t$ is in $K, h(t)=A(t)\left[h\left(t^{-}\right)\right]$and $h\left(t^{+}\right)=B(t)[h(t)]$, and the proof is complete.

Corollary 2. Let $V$ be in $O A I, W=E[V]$, and let $K, A$, and $B$ be as in Theorem 14. Let $p$ be in $X$, and let $h$ be the function determined in the proof of Theorem 14. 
Then if $t$ is in $S$, not in $K$, and $[c, t]$ intersects $K$, then

$$
h(t)=W(t, c)\left[\prod_{t(\sin K)}^{c}\left[W\left(s^{+}, c\right)^{-1} B(s) A(s) W\left(s^{-}, c\right)\right]\right][p] .
$$

Proof. Let $U_{1}$ and $U_{2}$ be as in the proof of Theorem 14. Let $T$ be a member of $O A$ such that if $[a, b]$ is an interval in $S$ and $q$ is in $X$, then

$$
T(b, a)[q]=(L, R) \int_{b}^{a} W(, c)^{-1}\left[U_{1}+U_{2}\right] W(, c)[q] .
$$

Now, by Theorem $1, h$ is given by $h(t)=W(t, c)\left[\Pi_{t} \Pi^{c}[I+T][p]\right]$. If $t$ is in $S$, not in $K$, and $[c, t]$ intersects $K$, then

$$
\prod_{t} \prod^{c}[I+T][p]=\prod_{(s \operatorname{in} K)}^{c}\left[\left[I+T\left(s^{+}, s\right)\right]\left[I+T\left(s, s^{-}\right)\right]\right][p] .
$$

But, if $s$ is in $K$,

$$
\begin{aligned}
T\left(s^{+}, s\right) & =W\left(s^{+}, c\right)^{-1} U_{2}\left(s^{+}, s\right) W(s, c) \\
& =W\left(s^{+}, c\right)^{-1}\left[B(s)-I-V\left(s^{+}, s\right)\right] W(s, c) \\
& =W\left(s^{+}, c\right)^{-1} B(s) W(s, c)-W\left(s^{+}, c\right)^{-1} W\left(s^{+}, c\right) \\
& =W\left(s^{+}, c\right)^{-1} B(s) W(s, c)-I .
\end{aligned}
$$

Similarly, if $s$ is in $K$,

$$
T\left(s, s^{-}\right)=W(s, c)^{-1} A(s) W\left(s^{-}, c\right)-I .
$$

This completes the proof.

ACKNOWLEDGEMENT. The author gratefully acknowledges the helpful recommendations of the referee with respect to Theorem 14 and Corollary 2 .

\section{REFERENCES}

1. W. A. Coppel, Stability and asymptotic behavior of differential equations, Heath, Boston, Mass., 1965. MR 32 \#7875.

2. F. R. Gantmacher, The theory of matrices, GITTL, Moscow, 1953; English transl. Vol. 2, Chelsea, New York, 1959. MR 16, 438; MR 21 \#6372c.

3. B. W. Helton, Integral equations and product integrals, Pacific J. Math. 16 (1966), 297-322. MR 32 \#6167.

4. J. V. Herod, Multiplicative inverses of solutions for Volterra-Stieltjes integral equations, Proc. Amer. Math. Soc. 22 (1969), 650-656.

5. J. S. Mac Nerney, Integral equations and semigroups, Illinois J. Math. 7 (1963), 148173. MR 26 \#1726.

6. —, A nonlinear integral operation, Illinois J. Math. 8 (1964), 621-638. MR 29 \#5082.

7. R. H. Martin, Jr., A bound for solutions of Volterra-Stieltjes integral equations, Proc. Amer. Math. Soc. 23 (1969), 506-512. MR 40 \#662.

8. - Bounds for solutions to a class of nonlinear integral equations (submitted for publication).

9. I. W. Neuberger, Continuous products and nonlinear integral equations, Pacific J. Math. 8 (1958), 529-549. MR 21 \#1509. 
10. C. C. Ross, Jr., On the multiplication of solutions of homogeneous linear differential systems, J. Math. Anal. Appl. 25 (1969), 266-271. MR 38 \#1311.

11. F. W. Stallard, Differential systems with interface conditions, Oak Ridge National Laboratory Publication \#1876 (Physics) 1955.

12. - Functions of bounded variation as solutions of differential systems, Proc. Amer. Math. Soc. 13 (1962), 366-373. MR 25 \#2276.

13. A. Zettl, Adjoint and self-adjoint boundary value problems with interface conditions, MRC Technical Report \#827, 1967.

Georgia Institute of Technology,

Atlanta, Georgia 30332 